\title{
Sintaxis del futuro: el proyecto de una Sudamérica real, posible e imaginada
}

\author{
Karina Vasquez*
}

Castro, Fernando Vale. Pensando um continente. A Revista Americana e a criação de um projeto cultural para a América do Sul. Rio de Janeiro: Editora Mauad, 2012.

En Pensando un continente..., Fernando Vale Castro se propone analizar la Revista Americana, una publicación que surge en las filas de Itamaraty — dirigida por los diplomáticos Araujo Jorge y Delgado de Carvalho, y el periodista Joaquín Viana - en Río de Janeiro durante la década comprendida entre 1909 y 1919. Contra la perspectiva que reduce este emprendimiento a un simple instrumento de divulgación de los lineamientos de Itamaraty, apenas como un mero reflejo o caja de resonancia de las principales posiciones del Barão de Rio Branco, el autor plantea la necesidad de comprender esta revista como una "comunidad argumentativa", donde convergen diversas opiniones que sin embargo comparten un objetivo general, ligado al mutuo acercamiento entre las distintas naciones sudamericanas y a la construcción de una estrategia diplomática capaz de garantizar en el continente la solución pa- cífica de conflictos en un contexto mundial caracterizado por una enorme incertidumbre e inestabilidad. Si, tal como sugiere el autor, toda revista puede ser entendida por la dialéctica entre producción y recepción, en este caso se propone priorizar " $a$ ótica dos produtores", entendiendo — en la línea de Skinner y Pocock - la producción de los discursos plasmados en la revista como "actos de habla" que, al mismo tiempo, surgen y modifican el contexto lingüístico en el que adquieren sentido e inteligibilidad.

Comprender estos discursos como "actos de habla" implica ponerlos en relación con sus contextos, y en el primer capítulo Vale Castro presenta las principales preocupaciones que atraviesan el contexto de creación de la revista. En este sentido, el autor articula un panorama que va desde las aceleradas transformaciones que permearon de manera general el último tercio del siglo XIX con las particularidades del contexto brasilero, signado por la proclamación de la República, las apuestas, esperanzas y desilusiones de la llamada "generación de 1870" en pos de un proyecto modernizador para el Brasil, y el afianzamiento de una dirección clara en la

* Doctora en Ciencias Sociales y Humanas por la Universidade de Qulimes (Argentina) y profesora en la Universidad de Quilmes y en la Universidad de Buenos Aires (UBA). Buenos Aires, Argentina. E-mail: kvasquez@unq.edu.ar. 
política exterior, a partir del nombramiento del Barão de Rio Branco en el ministerio de Itamaraty. Esta figura condensa un haz de problemas, entre los cuales el autor destaca conceptualmente dos que van a resultar particularmente relevantes en las reflexiones de la Revista Americana. Por un lado, la cuestión del establecimiento de los límites fronterizos y la unidad territorial, que si ya desde el imperio aparecen asociados al problema de la construcción del Estado, con el establecimiento de la República adquieren un importante rol material y simbólico en la consolidación de un proyecto de nación. Y si el Barão de Rio Branco va a ser la figura que encarne el éxito de la estrategia político-diplomática en la resolución de conflictos fronterizos, convirtiéndose así en el "ícone de um país, ao menos em tese, unido, estável e com visibilidade externa" (p. 37), es a partir de ella que la revista puede plantearse - a través de la problemática de las fronteras - la elaboración de un proyecto que valorice a la diplomacia, como agente cultural capaz de promover el conocimiento mutuo y de llevar a cabo las acciones/negociaciones que contribuyan al establecimiento de un equilibrio geopolítico, garante de la paz en épocas tumultuosas. Por otro lado, la segunda cuestión que va a resultar particularmente relevante en las páginas de la revista es el acercamiento de Itamaraty a EEUU - un proceso que había comenzado durante los últimos años del imperio y se afianza con la República - y la consiguiente adopción del panamericanismo, que va a constituir una de las principales características de la política del Barão —, adopción que parte del reconocimiento del ascenso de EEUU a potencia mundial y que aspira también a posicionar favorablemente a Brasil frente a Europa y al resto de las naciones sudamericanas. Caracterizado el contexto, el autor esboza las diferentes etapas que marcaron a este emprendimiento de larga duración. Así es que, siguiendo las sugerencias de Vale Castro, se pueden distinguir dos etapas, marcadas por sucesivas interrupciones: la primera, desde su creación en 1909 hasta la muerte del Barão de Rio Branco en 1912, momento en que se da la primera interrupción larga de la publicación, debida - entre otras cosas - a dificultades provocadas por la gran guerra con el abastecimiento de papel; la segunda comprende los cuatro números que salieron en 1915, con un perfil más comercial y popular, incorporando incluso un "Suplemento ilustrado" de contenido variado, y los últimos años de la revista (19171919), donde se retoma el formato anterior, buscando mantener como eje central de los artículos la problemática de la aproximación intelectual, política, económica y cultural de las naciones de América.

Después de esta presentación general de la revista, Vale Castro aborda en el capítulo 2 la problemática del pan-americanismo en la Revista Americana. Tal vez, sea este capítulo uno de los momentos centrales del libro, dado que la perspicacia analítica del autor permite aprehender a la revista como un emprendimiento plural y complejo que, si bien en líneas generales sostenía una posición afin al acercamiento a EEUU propiciado por Itamaraty, sin embargo acogió en sus páginas el debate, presentando también artículos 
que expresaron posiciones claramente contrarias a la orientación de la política externa brasilera. El autor analiza, en primer lugar, qué se entendía por esos años por "pan-americanismo" basado en la Doctrina Monroe, un intento de la política externa norteamericana de presentar a América Latina como un área subordinada a sus intereses económicos y políticos. El problema es que, si bien por motivos pragmáticos, el Barão de Rio Branco promovió un acercamiento a EEUU, no fue este el caso mayoritario del resto de los países sudamericanos, y este es un dato relevante a fin de visualizar los presupuestos comunes y los conflictos - explícitos o implícitos - que permearon la construcción de esta "comunidad argumentativa" que constituyó la revista. Así, si a principios del siglo XX, el debate sobre el alineamiento con EEUU, tiene al interior de la intelectualidad brasilera fuertes defensores - como Joaquim Nabuco - y detractores - como es el caso Oliveira Lima —, pareciera que ya para 1910 se produce cierta marginalización de esas posiciones críticas del panamericanismo, a tal punto que, en los primeros años de la revista este debate pareciera dividir, por un lado, a los intelectuales diplomáticos brasileros, ligados directamente a Itamaraty (como el propio Nabuco, Araripe Jr, Helio Lobo), que defienden una posición claramente favorable a la Doctrina Monroe y, por extensión, a la política norteamericana en el continente; y del otro, intelectuales latinoamericanos (el argentino Norberto Piñero, el chileno Macial Martínez, el venezolano Jacinto López, entre otros) que manifiestan más bien sus críticas a las aspiraciones he- gemónicas e intervenciones continentales de EEUU, planteando en algunos casos alternativas, como un ibero-americanismo o un pan-iberismo (alternativas que apostaban más bien a un estrechamiento de los vínculos con Europa, más que con EEUU). Ya en el segundo momento de la revista, después de 1915, hay una inflexión provocada mayormente por los efectos de la gran guerra, por la cual ya no hay espacio para posiciones contrarias a la aproximación entre América del Sur y Estados Unidos. En este sentido, los artículos analizados en esta etapa muestran cierta coincidencia en torno a una visión más favorable del panamericanismo. El autor señala que su recorrido por las dos etapas de la revista muestra un cierto redimensionamiento del concepto de monroísmo, que va desde la acérrima defensa de la Doctrina Monroe a una visión más crítica, que deja de lado la perspectiva intervencionista norteamericana, para poner en el énfasis en un discurso basado en un discurso basado en la cooperación, en la integración, en un modelo de pan-americanismo más directamente ligado los intereses de América del Sur.

En el tercer capítulo, Vale Castro plantea un análisis de algunos conceptos centrales que fueron objeto de discusiones y articularon debates en la revista - tales como la necesidad de promover el respeto a los principios del derecho público internacional, intervenciones en torno a los conceptos de soberanía y hegemonía, la cuestión de las fronteras como elemento primordial en la construcción de cualquier proyecto nacional, el debate en torno a la navegabilidad de los ríos - con el objetivo de mostrar cómo, más 
allá de los desacuerdos, la revista perseguía como objetivo central la elaboración de un "ideário americano, baseado no intercâmbio e na cooperação entre as naçóes americanas, capitaneadas pela diplomacia do continente" (p. 155). Desde la perspectiva de la revista, es justamente el cuerpo diplomático - entendido como una élite intelectual, relativamente independiente del poder políticoaquel sujeto capaz de llevar adelante tanto la construcción de una "moral sudamericana” para las relaciones internacionales, así como también crear las condiciones de un equilibrio continental que debiera servir de ejemplo a otras regiones del planeta. Lejos de pensar la trayectoria de la revista como una simple reflejo de las opiniones del Baráo de Rio Branco, este emprendimiento despliega toda una serie de intervenciones que apuntan a reflexionar sobre el papel que la diplomacia brasilera debería jugar - como sostiene Vale Castro - en la elaboración no tanto de un diagnóstico preciso, sino más bien de un pronóstico, "um projeto de futuro que deveria ser construído com uma América muito mais do que real, uma América possível, uma América imaginada" (p. 156). Que esa América posible fuera real, dependía de la acción de los intelectuales - en especial, diplomáticos - en pos del diálogo, la cooperación y el conocimiento mutuo entre naciones diversas, operaciones que la revista pretendió llevar a cabo no sólo a partir de la presentación de distintos argumentos y debates en la sección artículos, sino también a partir de otras secciones, aludidas en el libro, como las "Notas" y "Bibliografía", donde es posible visualizar una primera ten- tativa de crear un tejido cultural, que permitiera pensar el continente.

Sin duda, el esfuerzo analítico de Vale Castro ha permitido construir una imagen más rica, compleja y plural de la Revista Americana, que nos invita a reflexionar tanto sobre las nuevas representaciones de América que comienzan a surgir a principios de siglo, como también sobre los diversos roles que los intelectuales imaginan para sí frente a esas representaciones continentales, roles que motivan discursos, acciones, contactos, acercamientos mutuos y la aparición de redes que, en muchas ocasiones, sostuvieron o acompańaron emprendimientos comunes. Desde esta perspectiva, junto a los planteos de Skinner y Pocock, quizás hubiera sido útil incorporar también algunos otros aportes de la historia intelectual, en particular aquellos que proponen abordar las revistas como una "estructura de sociabilidad", en el sentido que esta noción ha sido definida en los trabajos de Jean-François Sirinelli y Michel Trebisch. ${ }^{1}$ En este sentido, si bien Vale Castro acota su análisis al espacio escrito y público de la revista (es decir, sabemos muy poco de las condiciones internas en torno a cómo se armaba el sumario, cómo se seleccionaban los

\footnotetext{
${ }^{1}$ Cfr. Sirinelli, Jean-François. Le hasard ou la nécessité? Une histoire en chantier: l'historie des intellectuels. Vigtième siècle. Revue d'histoire, n. 9, janvier-mars, p. 97-108, 1986; Trebitsch, Michel. Avant-propos : la chapelle, le clan et le microcosme; Racine, Nicoli y Trebitsch, Michel. Sociabilites Intellectueles. Lieux, Millieux, Reséaux. Cahiers de L'IHTP, n. 20, CNRS, París, p. 11-21; Pluet-Despatin; Jacqueline. Une contribution a l'histoire des intellectuals: les revues, en Les Cahiers de L' IHTP, n. 20, marzo de 1999, número especial "Sociabilites intellectuels: lieux, milieux, reseaux", p. 125-136.
} 
artículos, a partir de qué vínculos o redes llegaban las diversas intervenciones de autores latinoamericanos, cómo se contactaba a los autores, etc.), también restringe mayormente su análisis a un aspecto de la revista — aquel asociado directamente a la actividad diplomática - dejando en un segundo plano el análisis de aquellas intervenciones ligadas a la historia, a la literatura, y a la sociabilidad intelectual. Según refiere el autor, esta publicación contaba con una sección de "Notas", otra de "Bibliografía" y una dedicada a las "Revistas": hubiera sido muy útil analizar los libros y las revistas reseñadas, justamente para discernir qué tipo de sociabilidad, ampliada a la escena continental, proponía la Revista Americana. Al final de su texto, el autor nos presenta un índice de los artículos: el problema de este índice es que, si bien re- sulta útil el panorama general que ofrece al lector en pocas páginas, es un índice trunco, que eclipsa la "sintaxis" de la revista, dado que cada número no se reduce al listado de artículos. Tal como aparece en las fotos que acompañan al libro, en las ediciones de la revista hay intervenciones poéticas, comentarios, reseńas, notas de redacción, etc., en definitiva, textos y discursos - que junto con el formato que asumió cada número- definen de forma activa las estrategias por las cuales este emprendimiento se dirigió a sus diversos públicos. El análisis de Vale Castro complejiza, en muchos sentidos, la relación de la revista con Itamaraty; creo que hubiera sido deseable también visualizar de forma más precisa cómo la revista se construye como un espacio de sociabilidad para los intelectuales y escritores del continente. 